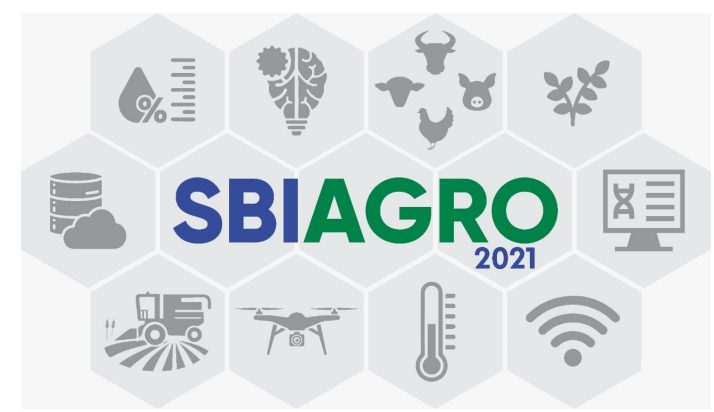

\section{Estudo comparativo entre Máquina de Vetores de Suporte e Rede Neural Convolucional na classificação do inseto Diaphorina citri}

\author{
Mário Lúcio G. de Q. Pierre Júnior ${ }^{1}$, José Leonardo dos S. Melo ${ }^{2}$, Marcelo P. de \\ Miranda $^{3}$, Michele Fúlvia Angelo ${ }^{4}$ \\ ${ }^{1}$ Instituto Federal de Educação, Ciência e Tecnologia Baiano \\ Senhor do Bonfim, Bahia, Brasil \\ ${ }^{2}$ Instituto Federal de Educação, Ciência e Tecnologia da Bahia \\ Euclides da Cunha, Bahia, Brasil \\ ${ }^{3}$ Fundo de Defesa da Citricultura \\ Araraquara, São Paulo, Brasil \\ ${ }^{4}$ Departamento de Ciências Exatas, Universidade Estadual de Feira de Santana \\ Feira de Santana, Bahia, Brasil \\ \{mario.junior\}@ifbaiano.edu.br, mfangelo@ecomp.uefs.br
}

\begin{abstract}
Diaphorina citri is the main propagator of Huanglongbing citrus disease. To control the problem, the insects are captured by adhesive traps and manually counted to adjust the insecticide dosage. Thus, this work aimed to measure and analyze the performance of the automatic classification of Diaphorina citri from Machine Learning approaches. For the training of the models, an image dataset was created and the Support Vector Machine (SVM) and Convolutional Neural Network (CNN) approaches were used. CNN, with Inception v3 model, presented an average of $99.37 \%$ accuracy in the final classification of Diaphorina citri, a significant result in relation to the $97.99 \%$ presented by SVM.
\end{abstract}

Resumo. O inseto Diaphorina citri é o principal propagador da doença dos citros Huanglongbing. Para o controle do problema, os insetos são capturados por armadilhas adesivas e contados manualmente, para ajuste da dosagem de inseticida. Assim, este trabalho teve como objetivo medir e analisar o desempenho da classificação automática do Diaphorina citri a partir de abordagens de Aprendizado de Máquina. Para o treinamento dos modelos foi criado um banco de imagens e utilizadas as abordagens de Máquina de Vetores de Suporte 
(SVM) e Rede Neural Convolucional (CNN). A CNN, com modelo Inception v3, apresentou em média $99.37 \%$ de acurácia na classificação final do Diaphorina citri, resultado significativo em relação aos $97.99 \%$ apresentado pela SVM.

\section{Introdução}

O Brasil é o maior fornecedor mundial de frutas cítricas in natura e outros subprodutos [Neves et al. 2010]. No entanto, apesar de toda sua importância, os problemas fitossanitários causam grandes perdas econômicas aos produtores e tornam mais difícil a exportação do suco de laranja brasileiro [Nava et al. 2007].

Uma das preocupações mais sérias do setor é o Huanglongbing (HLB), também conhecido como Greening, considerada a pior doença de citros. Ela tem como característica a clorose, entupimento dos vasos responsáveis por levar água e nutrientes da raiz para a copa da planta, causando a redução e perda da qualidade do fruto, deixando-o com sabor amargo [Halbert e Manjunath 2004].

Sem métodos de cura, o manejo da doença é realizado com inspeções para identificação, eliminação das plantas sintomáticas e o controle químico, que é a maneira mais utilizada pelo citricultor para reduzir a população do Diaphorina citri [Yamamoto et al. 2009], principal inseto propagador da bactéria causadora da doença do HLB.

O psilídeo Diaphorina citri é um pequeno inseto sugador que mede de 2 a $3 \mathrm{~mm}$ de comprimento, de cor cinza e manchas escuras nas asas [Gallo et al. 2002]. Devem ser realizadas pulverizações periódicas para o controle do inseto com o intuito de reduzir a probabilidade de ocorrência de novas infecções [Belasque Junior et al. 2009]. A tomada de decisão para a pulverização é realizada por meio do monitoramento. As armadilhas adesivas amarelas são utilizadas como forma de atração e captura de insetos e é um método de inspeção visual [Belasque Junior et al. 2009].

Um dos grandes problemas na utilização desse método de inspeção visual é que as armadilhas atraem outros insetos além do Diaphorina citri [Yamamoto et al. 2009]. Após dias de exposição no pomar, as armadilhas adesivas são retiradas e levadas para o laboratório. Através da identificação e contagem manual dos psilídeos encontrados, realizada por técnicos treinados, são feitos cálculos para se estimar a população da praga [Leonardo 2014].

O monitoramento é um importante componente do manejo integrado de pragas, pois indica o momento correto para a tomada de decisão, evitando aplicações desnecessárias de inseticidas e, consequentemente, o desequilíbrio biológico [Yamamoto et al. 2009]. Portanto, metodologias que automatizam esse processo de identificação e melhorem os resultados de acurácia são importantes para um processo eficiente de controle. Assim, o objetivo deste trabalho é medir e analisar o desempenho da classificação automática do inseto Diaphorina citri a partir de abordagens de Aprendizado de Máquina.

\section{Materiais e Métodos}

Os experimentos neste trabalho foram executados com as seguintes configurações de hardware e software: Processador Intel Corei7-7500U 2.70GHz; memória de 8GB; Disco 
rígido de 1TB; Placa de vídeo NVIDIA R GeForce R MX150 de 4GB; Sistema operacional Ubuntu 16.04 LTS; Python 2.7.12; Keras 2.2.0; TensorFlow 1.9.

Para o desenvolvimento foram necessárias as seguintes etapas: Criação do dataset de imagens das armadilhas adesivas; Classificação automática do inseto Diaphorina citri; e Avaliação do classificador. A seguir serão descritas cada uma das etapas.

\subsection{Criação do dataset de Imagens}

$\mathrm{O}$ dataset de imagens foi criado com amostras digitalizadas das armadilhas adesivas, disponibilizadas pelo Fundo de Defesa da Citricultura (FUNDECITRUS). O recorte dos insetos, nas imagens digitalizadas, foi feito manualmente utilizando o software ImageJ e resultando em 551 amostras do Diaphorina citri e 551 amostras de outros insetos, com tamanhos variados e posições distintas no quadrante do recorte, conforme apresentado na Figura 1.

O uso da quantidades iguais de amostras é para impedir o desbalanceamento, ou desequilíbrio na quantidade de amostras por classe, com objetivo de evitar problemas de generalização na classificação. Segundo Japkowic [Japkowicz e Stephen 2002], o desequilíbrio de classes pode ter um efeito prejudicial no treinamento de classificadores, afetando a convergência no treinamento e a generalização do modelo no conjunto de teste.

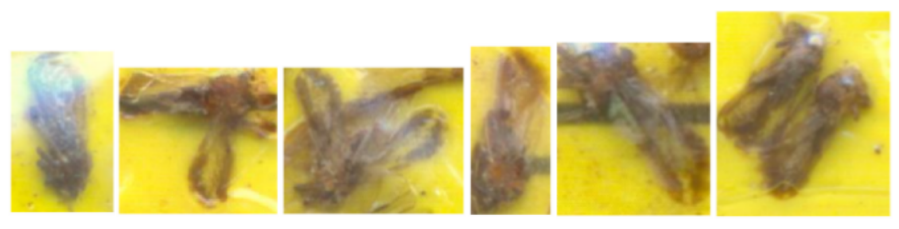

Figura 1. Recorte dos insetos

\subsubsection{Incremento do dataset}

Um dos métodos mais comuns para reduzir o super ajustamento durante o treinamento em aprendizado de máquina, aumentar a capacidade do classificador em generalizar e classificar padrões de testes que não foram utilizados no treinamento, é aumentar artificialmente o conjunto de imagens produzindo-se amostras transformadas a partir das imagens originais [Krizhevsky et al. 2012].

Para o aumento artificial das imagens foi utilizada a biblioteca Augmentor, resultando no aumento de 7.5 vezes do tamanho original do dataset, totalizando 8.354 amostras, divididas igualmente para Diaphorina citri e outros insetos nas dimensões de $224 \mathrm{x}$ 224 e 299 x 299 pixels, dimessões de entrada para as abordagens SVM e CNN, respectivamente.

Neste trabalho, o dataset de imagens foi dividido em $70 \%$ das amostras destinadas para o conjunto de desenvolvimento e $30 \%$ das amostras para o teste final. O conjunto de desenvolvimento foi subdividido em outros três conjuntos, com $90 \%$ destas imagens divididas em partes iguais para o treinamento e validação e os $10 \%$ restantes destinadas para os testes de parâmetros do conjunto de desenvolvimento. 


\subsection{Classificação automática do Diaphorina Citri}

Neste trabalho foi proposta a classificação do Diaphorina citri através de algoritmos de aprendizagem de máquina com as abordagens de Máquina de Vetores de Suporte e Rede Neural Convolucional. Os métodos serão descritos a seguir.

\subsubsection{Support Vector Machine (SVM)}

O SVM foi escolhido devido a sua ampla utilização em abordagens bens sucedidas de classificação de imagens [Boiman et al. 2008], [Chaplot et al. 2006], [Kotsia e Pitas 2006]. O SVM foi executado com sua configuração padrão e com kernel linear. Essas escolhas deveram-se a simplicidade de classificação desse núcleo e ao pequeno erro de generalização, o baixo custo computacional.

Nesta abordagem foram utilizados os algoritmos extratores de característica SIFT (Scale Invariant Feature Transform) [Lowe 2004] e o SURF (Speeded Up Robust Features) [Bay et al. 2008]. Para a implementação dos extratores foi criado um procedimento computacional que executou as tarefas de: ler os arquivos contendo os endereços das imagens, carregar cada imagem na memória, convertê-la para a escala de cinza, extrair suas características e salvar em arquivo o resultado dessas extrações, indexando-as de acordo com os rótulos: 1 para o Diaphorina citri e 0 para os outros insetos da base.

O método de anotação de características utilizado foi o Bolsa de Características (BoF - Bag-of-Features) [Tsai 2012] [Bosch et al. 2007]. A geração e representação dessas características pelo BoF segue a sequência: detecção de regiões ou pontos de interesse; descrição dos locais dessas regiões ou pontos de interesse; quantização dos descritores agrupando-os em palavras visuais para o livro de código (Codebook); contagem das palavras visuais específica do vocabulário visual para a construção do vetor de características BoF [Bosch et al. 2007].

Os livros de código foram criados usando o algoritmo de aprendizagem de máquina não-supervisionada Mini Batch K-Means. Esse algoritmo forma grupos (clusters) de coisas similares. Foi definida a quantidade de agrupamentos em 300 conjuntos, baseado no baseado no livro "Mastering Machine Learning with Scikit-Learn [Hackeling 2017]

Desta forma, foram criados três tipos distintos de livros de código. Um livro de código "Geral", que engloba tanto as características do Diaphorina citri quanto a dos outros insetos, agrupadas em 300 conjuntos. O segundo livro de código criado somente com as características do "Diaphorina citri", agrupadas em 300 conjuntos. E, no terceiro, foi criado um livro de código com 300 grupamentos de características das "Duas Classes", Diaphorina citri e outros insetos, totalizando 600 agrupamentos.

\subsubsection{Rede Neural Convolucional (CNN)}

Neste trabalho, utilizou-se a rede Inception v3, que é baseada na arquitetura GoogLeNet que venceu a competição ILSVRC (ImageNet Large-Scale Visual Recognition Challeng) em 2014. Esse modelo apresenta a sequência de módulos Inception A, B e C resultando 
em 42 camadas de rede. Embora apresente uma grande quantidade de camadas, o custo computacional é apenas cerca de 2,5 maior que o da GoogLeNet [Szegedy et al. 2016].

Os experimentos foram realizados utilizando parâmetros semelhantes aos da abordagem anterior. Foi definido o valor de 50 epochs de iterações do algoritmo e com 32 batch size, correspondente ao grupo de imagens selecionadas para o treinamento da rede. Definida a função categorical cross entropy para o cálculo do valor de erro e a função de ativação softmax para calcular as probabilidades de pertencimento de uma amostra para as duas classes definidas. Foi utilizado o banco de imagens redimensionadas para $299 \mathrm{x}$ 299, que é a dimensão de entrada para a rede Inception v3 e os algoritmo de otimização Adam [Kingma e Ba 2014] e RMSprop [Tieleman et al. 2012].

\subsection{Avaliação dos Classificadores}

Quando os modelos são desenvolvidos, seu desempenho é avaliado no conjunto de validação e é selecionado o modelo com o melhor desempenho para a aplicação das amostras de teste final. Neste trabalho, as medidas de avaliação do modelo utilizadas foram: acurácia, precisão, recall e F1-score.

Além destas medidas, foi aplicada a Validação Cruzada que é um método para avaliar a capacidade de generalização de um modelo. Foi utilizada a validação cruzada $k$-fold, com o $k=2$, que subdividiu o conjunto de treinamento em $k$ subconjuntos iguais, contendo amostras distribuídas aleatoriamente. Em seguida, $k$ iterações de etapas de treinamento e validação foram aplicadas usando diferentes subconjunto de dados para a validação e o outro subconjunto para o treinamento do modelo. Este processo foi repetido cinco vezes. Para cada iteração, uma nova subdivisão de validação e treinamento foi gerada.

Também foram aplicados testes estatísticos para analisar se existem diferenças nas técnicas aplicadas para cada algoritmo de classificação. Pois, embora o desempenho de diferentes classificadores possa ser mostrado como diferente em conjuntos de dados específicos, é necessário confirmar se as diferenças observadas são estatisticamente significativas e não meramente coincidentes. Com os valores de acurácia resultantes dos classificadores, foi aplicado o teste não paramétrico Friedman, que indica que as informações não apresentam um padrão de distribuição, usado para comparar múltiplos algoritmos em vários domínios. E o uso do teste de Nemenyi para comparar os resultados dos experimentos em pares, os classificadores [Japkowicz e Shah 2011].

\section{Resultados e Discussões}

Ao executar o algoritmo SVM o resultado da classificação do inseto foi de $50.08 \%$ de acurácia. Os valores de agrupamentos foram reduzidos, empiricamente, de 300 para 250, 100 e 50 clusters. Tendo os resultados: $51.56 \%$ de acurácia para 250 clusters; $80.40 \%$ para 100 clusters. A quantidade de 50 clusters apresentou o melhor resultado com $97.99 \%$ de acurácia para a classificação do Diaphorina citri em imagens digitalizadas.

O trabalho de Venugoban [Venugoban e Ramanan 2014] mostrou um estudo simplificado na variação do número de agrupamentos de bolsas de caracterísitcas, aplicado a um contexto específico, e serviu de base para a definição de testes de variação de agrupamentos realizados nesta pesquisa. Os resultados dos testes também mostraram que o valor de 50 agrupamentos resultou em um melhor desempenho na classificação. 
Definido em 50 clusters, o algoritmo de classificação foi novamente executado e desta vez sendo aplicado o procedimento de validação cruzada 2-fold $x 5$. Na Tabela 1 estão os valores resultantes das 5 execuções do SVM. Na sexta coluna estão os resultados de acurácia e desvio padrão, em função da variação da acurácia alcançada no procedimento de validação cruzada. O tempo médio de cada classificação foi de 6.20 minutos.

Tabela 1. Resultado da classificação utilizando SVM com validação cruzada

\begin{tabular}{|c|c|c|c|c|c|c|}
\hline Exec & Acurácia & Recall & Precision & F1-Score & Validação Cruzada & Tempo \\
\hline 1 & $97.99 \%$ & $98.80 \%$ & $97.23 \%$ & $98.01 \%$ & $94.05 \%(+/-0.16 \%)$ & $00: 06: 25$ \\
\hline 2 & $97.21 \%$ & $97.97 \%$ & $96.41 \%$ & $97.18 \%$ & $95.67 \%(+/-0.09 \%)$ & $00: 06: 10$ \\
\hline 3 & $97.57 \%$ & $98.36 \%$ & $96.78 \%$ & $97.57 \%$ & $94.49 \%(+/-0.19 \%)$ & $00: 06: 00$ \\
\hline 4 & $98.18 \%$ & $98.00 \%$ & $98.39 \%$ & $98.19 \%$ & $95.02 \%(+/-0.08 \%)$ & $00: 06: 40$ \\
\hline 5 & $98.07 \%$ & $98.19 \%$ & $97.99 \%$ & $98.09 \%$ & $95.53 \%(+/-0.11 \%)$ & $00: 06: 25$ \\
\hline
\end{tabular}

A Tabela 2 apresenta os valores de acurácia, da abordagem CNN, do processo de validação cruzada 2-fold $x 5$ com os otimizadores Adam e RMSProp. Um ponto que chama atenção nos dados é o tempo de execução do algoritmo que apresentou em média 26 horas de execução, aumento significativo de execução em relação ao SVM. Apesar do aumento do custo computacional, o modelo Inception apresentou números importantes com $99.82 \%$, na primeira execução com o RMSProp, em relação a taxa de acurácia tanto no processo de validação cruzada como também na generalização do modelo.

Tabela 2. Resultados do treinamento com Inception

\begin{tabular}{|c|c|c|c|c|}
\cline { 2 - 5 } \multicolumn{1}{c|}{} & \multicolumn{2}{c|}{ ADAM } & \multicolumn{2}{c|}{ RMSProp } \\
\hline Execução & Validação Cruzada & Tempo & Validação Cruzada & Tempo \\
\hline 1 & $99.48 \%(+/-0.12 \%)$ & $26: 06: 00$ & $99.82 \%(+/-0.06 \%)$ & $25: 56: 00$ \\
\hline 2 & $99.80 \%(+/-0.08 \%)$ & $26: 00: 00$ & $98.67 \%(+/-0.10 \%)$ & $26: 16: 00$ \\
\hline 3 & $99.78 \%(+/-0.10 \%)$ & $25: 57: 00$ & $97.47 \%(+/-0.12 \%)$ & $26: 01: 00$ \\
\hline 4 & $99.84 \%(+/-0.05 \%)$ & $26: 03: 00$ & $98.35 \%(+/-0.24 \%)$ & $26: 00: 00$ \\
\hline 5 & $99.56 \%(+/-0.15 \%)$ & $26: 15: 00$ & $98.13 \%(+/-0.19 \%)$ & $26: 06: 00$ \\
\hline
\end{tabular}

A Tabela 3 apresenta os resultados de generalização do modelo Inception com suas medidas de avaliação para o conjunto de amostras de teste de parâmetros, com a aplicação dos otimizadores Adam e RMSprop. Os resultados de acurácia apresentaram valores de acertos próximos para os dois otimizadores, porém com o maior valor de acurácia sendo apresentado com o otimizador RMSprop na primeira e na quinta execução do processo de validação cruzada com $99.82 \%$, consequentemente apresentando poucos erros na classificação do Diaphorina citri. O resultado da matriz de confusão, com dados de testes de parâmetros, previu incorretamente somente uma amostra de diaphorina citri como sendo outros insetos e classificou corretamente as amostras dos outros insetos.

Para analisar se existem diferenças nas técnicas aplicadas a cada algoritmo de classificação, foi necessário avaliar os resultados através de testes estatísticos. É necessário confirmar se as diferenças observadas foram estatisticamente significativas ou não meramente coincidentes. Assim, o teste verificou a diferença dos resultados dos classificadores quanto a questão de significância dos resultados obtidos. 
Tabela 3. Resultados da generalização do modelo Inception com amostras para ajustes de parâmetros com os otimizados ADAM e RMSProp.

\begin{tabular}{|c|c|c|c|c|c|c|c|}
\hline \multicolumn{4}{|c|}{ ADAM } & \multicolumn{4}{c|}{ RMSProp } \\
\hline Acurácia & Recall & Precision & F1-Score & Acurácia & Recall & Precision & F1-Score \\
\hline $99.48 \%$ & $99.31 \%$ & $99.65 \%$ & $99.48 \%$ & $99.82 \%$ & $100 \%$ & $99.65 \%$ & $99.82 \%$ \\
\hline $99.66 \%$ & $99.66 \%$ & $99.66 \%$ & $99.66 \%$ & $99.66 \%$ & $100 \%$ & $99.32 \%$ & $99.66 \%$ \\
\hline $99.48 \%$ & $99.31 \%$ & $99.65 \%$ & $99.48 \%$ & $99.49 \%$ & $99.66 \%$ & $99.32 \%$ & $99.49 \%$ \\
\hline $99.66 \%$ & $99.32 \%$ & $100 \%$ & $99.66 \%$ & $99.49 \%$ & $98.98 \%$ & $100 \%$ & $99.49 \%$ \\
\hline $99.14 \%$ & $98.98 \%$ & $99.32 \%$ & $99.15 \%$ & $99.82 \%$ & $100 \%$ & $99.65 \%$ & $99.82 \%$ \\
\hline
\end{tabular}

$\mathrm{Na}$ avaliação dos resultados, foi aplicado o teste não paramétrico de Friedman. O resultado do teste apresentou o $p$-value, que representa a probabilidade de significância, de 0.04057 . Como o valor de significância de 0.04057 é menor que o valor de confiança estabelecido em 0.05 , foi rejeitada a hipótese nula $H_{0}$ que afirmaria serem iguais os classificadores, não apresentando diferença significativa em seus resultados. Ao ser rejeitada a hipótese nula, fez-se necessária a aplicação de um teste Post Hoc para o teste de Friedman.

Para o Post Hoc foi utilizado o teste de Nemenyi que compara os algoritmos e determina onde está a diferença entre eles [Japkowicz e Shah 2011]. No teste de $\mathrm{Ne}$ menyi, a comparação entre o algoritmo Inception com otimizador Adam x Inception com RMSProp não apresentou diferença significativa, com resultado maior que 0.05. Porém, a comparação entre o algoritmo Inception com o Adam x SVM teve como resultado o valor 0.004605 e o algoritmo Inception com RMSProp x SVM com o valor de 0.001291 , apresentou diferença significativa entre o modelo Inception com os otimizadores Adam e RMSprop e a metodologia SVM.

Demonstrando maior confiabilidade na classificação correta do inseto Diaphorina citri, foi selecionada para a execução final a arquitetura Inception. Para esta execução final foram utilizadas as amostras de imagens do conjunto de teste, correspondente a $30 \%$ das amostras do banco de imagem, que ainda não haviam sido utilizadas em nenhum procedimento anterior deste trabalho. Ao aplicar o conjunto com amostras de teste ao modelo Inception foi obtida uma média de acurácia de $99.51 \%$ na validação cruzada e $99.37 \%$ na validação com o resultado da generalização do modelo com o conjunto de teste final, conforme Tabela 4.

Os modelos de aprendizagem de máquina podem ser salvos durante e após o final do treinamento, evitando assim longos períodos de treinamento e podendo esse modelo ser compartilhado. Desta forma, a execução dos modelos no teste final teve a média de tempo de 33.03 minutos, conforme Tabela 4.

Analisando-se os resultados apresentados por [Shen et al. 2018] e [Liu et al. 2016], que também utilizaram aprendizado profundo para classificação e identificação de insetos, é possível constatar que o presente trabalho, apesar de não ter o mesmo inseto como objeto de pesquisa, apresentou a média final de acurácia de $99.51 \%$ na validação cruzada e $99.37 \%$ na validação do modelo, sendo estes resultados superiores aos obtidos nos trabalhos citados. 
Tabela 4. Resultado final do treinamento com o algoritmo Inception.

\begin{tabular}{|c|c|c|c|c|c|c|}
\hline Exec & Acurácia & Recall & Precision & F1-Score & Validação Cruzada & Tempo \\
\hline 1 & $99.12 \%$ & $99.44 \%$ & $98.80 \%$ & $99.12 \%$ & $99.28 \%(+/-0.08 \%)$ & $00: 34: 55$ \\
\hline 2 & $99.32 \%$ & $99.60 \%$ & $99.04 \%$ & $99.32 \%$ & $99.48 \%(+/-0.11 \%)$ & $00: 30: 40$ \\
\hline 3 & $99.56 \%$ & $99.68 \%$ & $99.44 \%$ & $99.56 \%$ & $99.64 \%(+/-0.13 \%)$ & $00: 32: 17$ \\
\hline 4 & $99.32 \%$ & $99.60 \%$ & $99.04 \%$ & $99.32 \%$ & $99.48 \%(+/-0.09 \%)$ & $00: 33: 01$ \\
\hline 5 & $99.44 \%$ & $99.60 \%$ & $99.28 \%$ & $99.44 \%$ & $99.68 \%(+/-0.27 \%)$ & $00: 34: 23$ \\
\hline
\end{tabular}

\section{Conclusão}

Este trabalho teve como objetivo medir e analisar o desempenho da classificação automática do inseto Diaphorina citri a partir de abordagens de Aprendizado de Máquina. Para isso foram utilizadas uma SVM e uma CNN. A CNN com a arquitetura de rede Inception apresentou os melhores valores de acurácia e menor quantidade de erros na classificação do Diaphorina citri. Por esse motivo, foi aplicado ao modelo o teste final de generalização que resultou em uma média de acurácia de $99.51 \%$ na validação cruzada e $99.37 \%$ na validação do modelo com o conjunto de teste final.

Como melhorias para esta pesquisa, poderá ser desenvolvido um algoritmo de processamento de imagens para a extração de amostras dos insetos contidos nas armadilhas adesivas, de maneira que automatize a criação do dataset de imagem. E também, aprimorar o algoritmo $\mathrm{CNN}$, utilizando outros parâmetros de otimização, objetivando reduzir o custo computacional. Poderão também ser experimentadas outras arquiteturas de CNN.

\section{Referências}

Bay, H., Ess, A., Tuytelaars, T., e Van Gool, L. (2008). Speeded-up robust features (surf). Computer vision and image understanding, 110(3):346-359.

Belasque Junior, J., Bergamin Filho, A., Bassanezi, R. B., Barbosa, J. C., Fernandes, N. G., Yamamoto, P. T., Lopes, S. A., Machado, M. A., Leite Junior, R. P., Ayres, A. J., et al. (2009). Base científica para a erradicação de plantas sintomáticas e assintomáticas de huanglongbing (hlb, greening) visando o controle efetivo da doença. Tropical Plant Pathology, 34(3):137-145.

Boiman, O., Shechtman, E., e Irani, M. (2008). In defense of nearest-neighbor based image classification. In 2008 IEEE Conference on Computer Vision and Pattern Recognition, pages 1-8. IEEE.

Bosch, A., Munoz, X., e Marti, R. (2007). Which is the best way to organize/classify images by content? Image and vision computing, 25(6):778-791.

Chaplot, S., Patnaik, L. M., e Jagannathan, N. (2006). Classification of magnetic resonance brain images using wavelets as input to support vector machine and neural network. Biomedical signal processing and control, 1(1):86-92.

Gallo, D., Nakano, O., Silveira Neto, S., Carvalho, R. P. L., e Baptista, G. C. d. (2002). Entomologia agrícola. Fundação de Estudos Agrários Luiz de Queiroz, 632.7 edition.

Hackeling, G. (2017). Mastering Machine Learning with scikit-learn. Packt Publishing Ltd. 
Halbert, S. E. e Manjunath, K. L. (2004). Asian citrus psyllids (sternorrhyncha: Psyllidae) and greening disease of citrus: a literature review and assessment of risk in florida. Florida entomologist, 87(3):330-353.

Japkowicz, N. e Shah, M. (2011). Evaluating learning algorithms: a classification perspective. Cambridge University Press.

Japkowicz, N. e Stephen, S. (2002). The class imbalance problem: A systematic study. Intelligent data analysis, 6(5):429-449.

Kingma, D. P. e Ba, J. (2014). Adam: A method for stochastic optimization. arXiv preprint arXiv:1412.6980.

Kotsia, I. e Pitas, I. (2006). Facial expression recognition in image sequences using geometric deformation features and support vector machines. IEEE transactions on image processing, 16(1):172-187.

Krizhevsky, A., Sutskever, I., e Hinton, G. E. (2012). Imagenet classification with deep convolutional neural networks. Advances in neural information processing systems, 25:1097-1105.

Leonardo, A. (2014). Otimização da leitura de cartão adesivo amarelo para o monitoramento de adultos de diaphorina citri kuwayama (hemiptera: Liviidae). Master's thesis, Fundo de Defesa da Citricultura.

Liu, Z., Gao, J., Yang, G., Zhang, H., e He, Y. (2016). Localization and classification of paddy field pests using a saliency map and deep convolutional neural network. Scientific reports, 6(1):1-12.

Lowe, D. G. (2004). Distinctive image features from scale-invariant keypoints. International journal of computer vision, 60(2):91-110.

Nava, D., Torres, M., Rodrigues, M., Bento, J., e Parra, J. (2007). Biology of diaphorina citri (hem., psyllidae) on different hosts and at different temperatures. Journal of Applied Entomology, 131(9-10):709-715.

Neves, M. F., Trombin, V., Milan, P., Lopes, F., Cressoni, F., e Kalaki, R. (2010). O retrato da citricultura brasileira. Ribeirão Preto: CitrusBR, page 137.

Shen, Y., Zhou, H., Li, J., Jian, F., e Jayas, D. S. (2018). Detection of stored-grain insects using deep learning. Computers and Electronics in Agriculture, 145:319-325.

Szegedy, C., Vanhoucke, V., Ioffe, S., Shlens, J., e Wojna, Z. (2016). Rethinking the inception architecture for computer vision. In Proceedings of the IEEE conference on computer vision and pattern recognition, pages 2818-2826.

Tieleman, T., Hinton, G., et al. (2012). Lecture 6.5-rmsprop: Divide the gradient by a running average of its recent magnitude. COURSERA: Neural networks for machine learning, 4(2):26-31.

Tsai, C.-F. (2012). Bag-of-words representation in image annotation: A review. International Scholarly Research Notices, 2012.

Venugoban, K. e Ramanan, A. (2014). Image classification of paddy field insect pests using gradient-based features. International Journal of Machine Learning and Computing, 4(1):1. 
Yamamoto, P. T., Fellipe, M. R., Sanches, A. L., Coelho, J. H., Garbim, L. F., e Ximenes, N. L. (2009). Eficácia de inseticidas para o manejo de diaphorina citri kuwayama (hemiptera: Psyllidae) em citros. BioAssay, 4. 